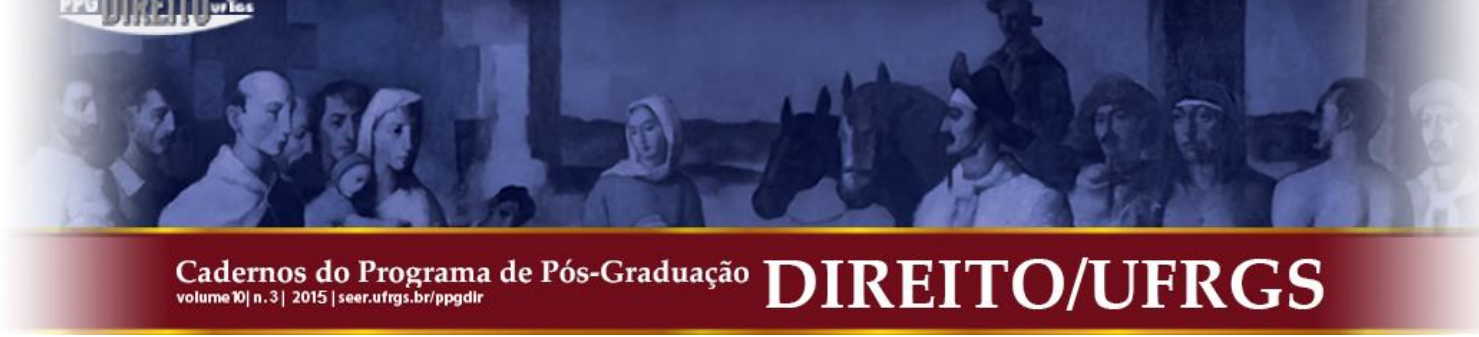

\title{
RÉFLEXIONS SUR LES PARTITIONS DU CONTRAT DE LOCATION DANS LES PRODUCTIONS DES GLOSSATEURS
}

\author{
REFLEXÕES SOBRE AS DIVISÕES DO CONTRATO DE LOCAÇÃO NOS TRABALHOS \\ DOS GLOSADORES
}

\author{
THOUGHTS ABOUT THE DIVISIONS OF THE CONTRACT OF LETTING AND HIRING \\ IN GLOSSATORS' WORKS
}

\section{Audrey Marie Dauchy*}

\begin{abstract}
RESUMO: Os glosadores, nos séculos XII e XIII, construíram a divisão do contrato de locação, locatio conductio, e revelaram seus aspectos de prestação de serviços e mesmo de locação de obra ou empreitada. Essa construção, no entanto, não é nem linear e nem sempre explícita. Alguns autores evocam unicamente a locação de coisas, o que coloca em questão o significado do seu silêncio. Alguns outros descreveram as diversas locações, mas com mais força em uma definição centrada sobre a locação de coisas. As relações entre as diferentes locações podem agora somente ser objeto de hipóteses. Outras fontes ainda mostram uma divisão clara do contrato entre a locação de coisas e a prestação de serviços, mas apresentam uma certa ambiguidade quanto à locação de obra ou empreitada. Os aspectos explícitos da divisão do contrato se revestem ainda de algo misterioso, porque eles remetem às questões da relação entre a coisa e a pessoa, e a identificação da pessoa aos serviços que ela presta.
\end{abstract}

Palavras-Chave: Locatio conductio. Contrato de locação. Locação de coisas. Prestação de serviços. Locação de obra ou empreitada. Trabalho. Pessoa. Glosadores.
RESUME : Les glossateurs, aux XII et XIII siècles, ont construit la division du contrat de location, locatio conductio, et ont révélé ses aspects de location de services, et même de location d'ouvrage. Cette construction, toutefois, n'est ni linéaire, $n i$ toujours explicite. Certains auteurs évoquent uniquement la location de chose, ce qui pose la question de la signification de leur silence. D'autres font état de plusieurs locations, mais présentent une définition centrée sur la location de chose. Les relations entre les différentes locations peuvent alors seulement faire l'objet d'hypothèses. D'autres sources encore montrent une partition claire du contrat entre la location de chose et la location de services, mais réservent un sort ambigu a la location d'ouvrage. Les aspects explicites de la division du contrat revêtent aussi une part de mystère puisqu'ils renvoient aux questions de la relation entre la chose et la personne, et de l'identification de la personne aux services qu'elle rend.

MotS-CLÉS : Locatio conductio. Contrat de location. Location de chose. Location de services. Location d'ouvrage. Travail. Personne. Glossateurs.
ABSTRACT: Glossators, in the 12th and 13th centuries, built the division of the contract of letting and hiring, locatio conductio, and revealed its aspects regarding the letting and hiring of services, and even the letting and hiring of work. This construction, however, is neither linear, nor always explicit. Some authors only mention the letting of a thing, which raises the question of the meaning of their silence. Some others describe several types of letting and hiring, but set out a definition focused on the letting of a thing. In this case, we can only make assumptions on the relationships between the different types of letting and hiring. Some other sources show a clear division of the contract between the letting of $a$ thing and the letting of services, but deal ambiguously with the letting of work. There is also a part of mystery remaining in the explicit aspects of the division of the contract, since they refer to such issues as the relationships between the thing and the person, and the identification of a person with the services provided.

KEYWORDS: Locatio conductio. Contract of letting and hiring. Letting and hiring of things. Letting and hiring of services. Letting and hiring of work. Labour. Person. Glossators.

SOMMAIRE : Introduction. 1. Les présentations unitaires du contrat, reflets d'une conception unitaire ? 1.1. Les présentations en apparence exclusivement fondées sur l'usage de la chose. 1.2. Les présentations fondées sur l'usage de la chose, ouvertes à d'autres locations. 2. Les déclinaisons du contrat de location. 2.1. Les cas explicites de bipartition du contrat : la chose et les services, la chose et la personne. 2.2. Au-delà de la bipartition du contrat ? Sources et bibliographie.

* Doutoranda em História do Direito pela Université Panthéon-Assas Paris II, França. Pesquisadora visitante no Max-Planck-Institut für europäische Rechtsgeschichte (MPIeR), Alemanha. Bolsista do Institut FrancoAllemand/Sciences Historiques et Sociales (IFRA/SHS). Mestre em História do Direito pela Université Panthéon-Assas Paris II, França. 


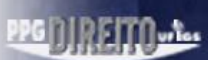

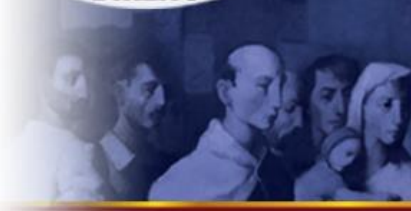

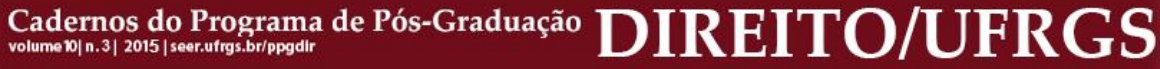

\section{INTRODUCTION}

Les expressions locatio rei, location d'une chose, locatio operarum, location de services, et de locatio operis (faciendi), location d'un ouvrage (à faire), permettent d'identifier trois branches constituant le contrat de louage.

Ces expressions ne sont pas employées en tant que telles dans les compilations justiniennes. Elles ne leur sont pas pour autant tout à fait étrangères, puisqu'une «chose » ou des « choses », un « service » ou des « services », et un « ouvrage » sont dits être loués, ou pris en location. Dans le titre du Digeste relatif à la locatio conductio, il est ainsi question de la solidarité des preneurs à l'égard de la chose louée ${ }^{1}$, de la jouissance de la chose prise en location $^{2}$, de la distinction entre les genres de choses louées ${ }^{3}$, ou encore du préjudice subi en raison de la chose prise en location ${ }^{4}$. Le terme service, au singulier ou au pluriel, est associé à la location à propos de la prise en location des services d'un esclave ${ }^{5}$, sont aussi développés les cas d'un secrétaire qui loue ses services ${ }^{6}$, et d'un artisan louant son service ${ }^{7}$; en outre, quelqu'un qui a loué ses services doit recevoir la rémunération de toute la durée de la location, s'il ne dépend pas de lui qu'il en ait moins répondu ${ }^{8}$. Enfin, le risque au regard de l'ouvrage loué est en principe à la charge du preneur jusqu'à ce que le bailleur approuve l'ouvrage ${ }^{9} ;$ l'expression «j'ai loué un ouvrage à faire » est employée à propos de l'action du bailleur en cas de réalisation défectueuse de l'ouvrage ${ }^{10}$, le délai d'achèvement de l'ouvrage est évoqué «dans la location d'ouvrage ${ }^{11}$, et lorsqu'une personne a loué un ouvrage pour une rémunération certaine, l'estimation du coût de construction de l'ouvrage ne peut dépasser le coût effectif ${ }^{12}$.

Ces références au louage de choses, de service ou d'ouvrage ne doivent cependant pas égarer le lecteur : à aucun moment, le contrat de louage ne fait l'objet d'une partition

${ }^{1}$ ULPIANUS. Libro trigesimo secundo ad edictum, D., 19, 2, 13, 9.

${ }^{2}$ Ibid. D., 19, 2, 15, 2.

${ }^{3}$ ALFENUS. Libro quinto a Paulo epitomarum, D., 19, 2, 31.

${ }^{4}$ PAULUS. Libro vicesimo secundo ad edictum, D., 19, 2, 45, 1.

${ }^{5}$ ULPIANUS. op. cit., D., 19, 2, 9, 1.

${ }^{6}$ Ibid. D., 19, 2, 19, 9.

${ }^{7}$ PAULUS. Libro trigesimo quarto ad edictum, D., 19, 2, 22, 2.

${ }^{8}$ ID. Libro singulari regularum, D., 19, 2, 38, pr.

${ }^{9}$ FLORENTINUS. Libro septimo institutionum, D., 19, 2, 36.

${ }^{10}$ IAVOLENUS. Libro undecimo epistularum, D., 19, 2, 51, 1.

${ }^{11}$ LABEO. Libro quarto posteriorum a Iavoleno epitomatorum, D., 19, 2, 58, 1.

${ }^{12}$ ID. Libro quinto posteriorum a Iavoleno epitomatorum, D., 19, 2, 60, 4. 


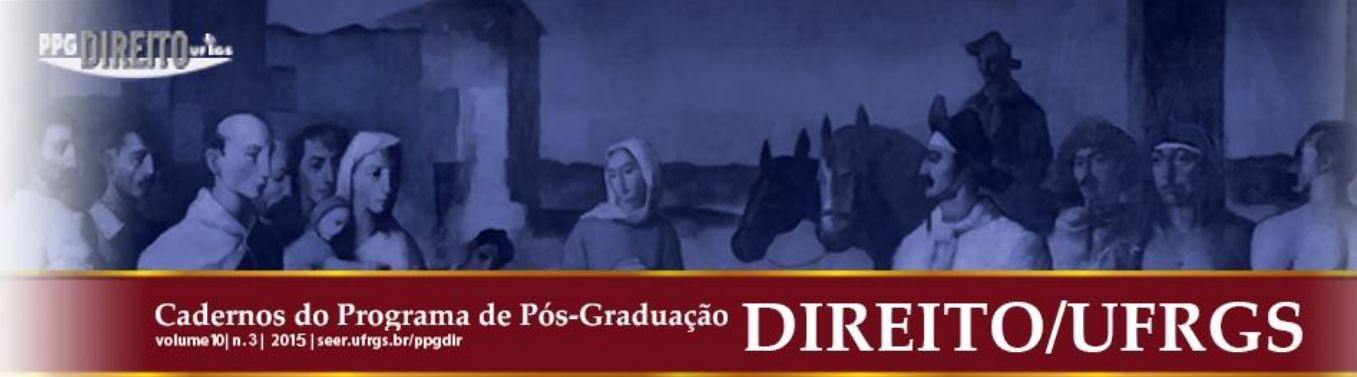

explicite. Plusieurs théories sur la nature du contrat de locatio conductio ont été avancées au $\mathrm{XX}^{\mathrm{e}}$ siècle, selon lesquelles le contrat aurait pu être considéré par les juristes romains comme unitaire, ou implicitement tripartite. Au-delà de cette question, il peut être simplement constaté que le contrat ne fait pas l'objet dans les compilations du $\mathrm{VI}^{\mathrm{e}}$ siècle d'une construction juridique opérant son partage.

À l'opposé, la construction du contrat en trois parties est marquée par une étape significative à la fin du XVII ${ }^{\mathrm{e}}$ et au début du XVIII ${ }^{\mathrm{e}}$ siècle, sous la plume du juriste néerlandais Johannes Voet. Ce dernier distingue, au titre de son commentaire sur les Pandectes concernant la locatio conductio, plusieurs types de contrats de louage. Sont ainsi différenciés, au sujet du louage, l'usage de choses et les services ${ }^{13}$, puis, au-delà de ces deux possibilités, l'ouvrage peut être loué ${ }^{14}$. L'usage de choses, les services et l'ouvrage sont ainsi identifiés et considérés comme pouvant être objets de location. Il peut être remarqué que Johannes Voet n'emploie pas la terminologie précise locatio rei, locatio operarum, locatio operis. Comme le signale Filippo Ranieri ${ }^{15}$, Johannes Gottlieb Heineccius utilise en revanche cette terminologie dans des développements consacrés au contrat ${ }^{16}$, tout en continuant à définir le contrat de louage comme un contrat consensuel d'usage de la chose ou de service, selon une vision bipartite ${ }^{17}$. L'histoire de la construction tripartite du contrat trouve enfin un point d'aboutissement avec

\footnotetext{
${ }^{13}$ VOET, JOHANNES. Com. ad D., 19, 2, n 6: «Praeter usum rerum elocantur et operae, tam liberorum hominum quam servorum, mercenariae, non aliae » [1ère éd., Leiden, 1698-1704], (Commentarius ad Pandectas, HagaeComitum, 1707, p. 827).

${ }^{14}$ ID. Com. ad D., 19, 2, n $\mathrm{n}^{\circ} 33$ : «Praeter usum et operas, praecipua locationis objecta, etiam opus locari potest » (op. cit., p. 838).

15 RANIERI, Filippo. «Dienstleistungsverträge: Rechtsgeschichte und die italienische Erfahrung». In: ZIMMERMANN, R. (ED.). Service contracts. Gesellschaft für Rechtsvergleichung. (Rechtsvergleichung und Rechtsvereinheitlichung, 15). Tübingen: Mohr Siebeck, 2010, p. 15.

16 HEINECCIUS, JOHANNES GOTTLIEB. Elementa juris civilis secundum ordinem Institutionum commoda auditoribus methodo, 3, 25, §. 917: «Est ergo locatio conductio vel rerum, quando rei usus, vel operarum, quando operae illiberales praestantur, vel operis, quando opus conficitur pro certa mercede » [1ère éd., Amsterdam, 1725], (éd. Giessae, ca. 1750, p. 417).

17 ID. Elementa juris civilis secundum ordinem Institutionum, 3, 25, §. 916 : «Alter contractus, qui consensu initur, est locatio conductio, quae est contractus consensualis de usu rei ad certum tempus, vel opera pro certa mercede praestandis » (op.cit., p. 417-418).
} 
chose est reçue en usage ${ }^{26}$. Il est fait dans la Lectura Vindobonensis une plus grande différence que dans la Summa Justiniani est in hoc opere entre les situations respectives du bailleur et du preneur. L'objectif de permettre l'usage de la chose y est ainsi uniquement marqué à l'égard du bailleur (ad usum), tandis que le rôle du preneur recevant la chose est davantage passif (in usum). Ces deux sources ont en commun d'être des ouvrages rédigés à l'aube du développement de l'école des glossateurs, et d'être une somme et une glose aux Institutes, elles-mêmes condensées. Faut-il en déduire que cette présentation exclusivement unitaire est le fait d'une pensée naissante, ou l'expression d'un impératif de concision?

La Summa Vindobonensis, que la Lectura du même nom a pour vocation de compléter, est plus ancienne encore que cette dernière. Il y est pourtant clairement énoncé une bipartition du contrat dans le cadre d'une définition : la location, selon l'auteur de la Summa Vindobonensis, se distingue ainsi par la concession de l'usage d'une chose ou de services contre une rémunération ${ }^{27}$. Cette somme, qui aurait pu être écrite par Irnerius, un élève de Martinus ou encore un contemporain des Quattuor doctores selon différentes hypothèses avancées $^{28}$, ne fait aucun mystère d'une partition du contrat entre la location de chose ou de services. L'exemple de la Summa Vindobonensis vient donc s'opposer à l'idée selon laquelle les premiers ouvrages des glossateurs livreraient des définitions strictement unitaires du contrat. Dans ces conditions, le caractère concis de la Lectura Vindobonensis pourrait expliquer, bien plus que sa rédaction précoce, sa définition exclusivement centrée sur l'usage de la chose. Il faut ajouter à cela que l'auteur de la Lectura Vindobonensis, après avoir fait état d'une définition exclusivement unitaire, ne se concentre pas uniquement sur des cas relatifs à la location de chose. Il évoque les exemples du foulon et du raccommodeur, en précisant que leurs activités ne feraient pas l'objet d'une location si la rémunération n'était pas établie immédiatement ${ }^{29}$. L'action de fouler ou de raccommoder des vêtements pourrait

\footnotetext{
${ }^{26}$ Lectura Vindobonensis, 24, $\mathrm{V}^{\circ}$ Locatio : «Locatio constat in re ad usum data, cum mercedis diffinitione conductio vero constat, cum res in usum recipitur cum constituta mercede » (éd. PALMIERI, J.B. Wernerii summa Institutionum cum glossis Martini, Bulgari, Alberici aliorumque. Bibliotheca Iuridica Medii Aevi. t. I. Additiones. Bononiae, 1914, p. 128).

27 Summa Vindobonensis, 24: «Est autem locatio cum usum rei mee, vel operas meas pro mercede tibi concedo» (éd. PALMIERI, J.B. Wernerii summa Institutionum cum glossis Martini, Bulgari, Alberici aliorumque. Bibliotheca Iuridica Medii Aevi. t. I. Additiones. Bononiae, 1914, p. 128).

${ }^{28}$ LANGE, Hermann. Römisches Recht im Mittelalter. t. I. Die Glossatoren. op. cit., §. 53, p. 435.

${ }^{29}$ Lectura Vindobonensis, 24, $\mathrm{V}^{\mathrm{o}} \mathrm{Cum}$ : «Si fulloni polienda, idest plananda vel ornanda, curandave, aut sarcinatori sarcienda vestimenta quis dederit, nulla statim mercede constituta, sed postea tantum daturus quantum inter eos convenerit, non proprie locatio et conductio contrahi intelligitur, quia certa merces non est constituta, 


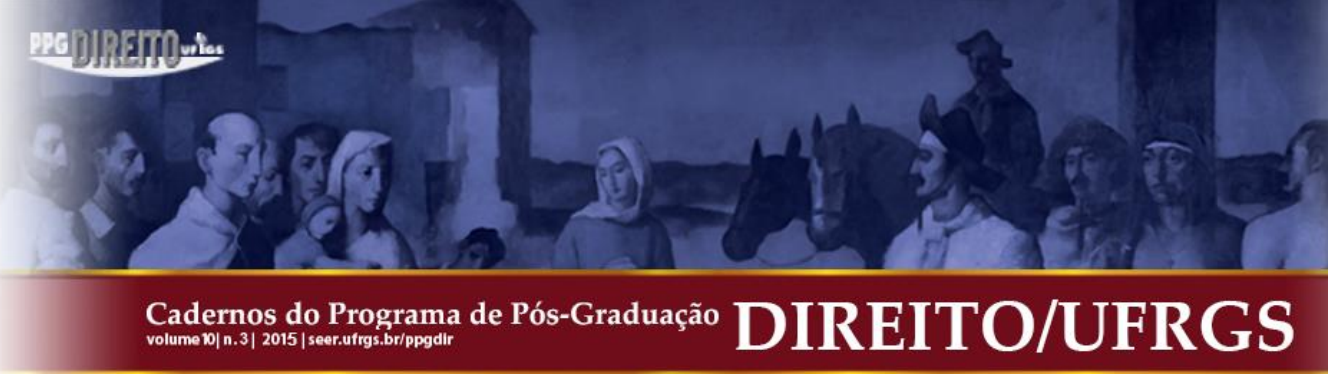

donc donner lieu à une location dans le cas d'une fixation immédiate de la rémunération. Ces exemples rappellent la casuistique des compilations justiniennes, et ne présentent en euxmêmes aucune nouveauté. Ils ont toutefois la vertu de montrer qu'une même source peut livrer une définition strictement unitaire, et présenter des exemples qui ne sont pas nécessairement ceux de la location de chose.

$\mathrm{Au}$ soutien de l'argument de la concision, d'autres sources plus tardives, qui appartiennent au genre des sommes aux Institutes et qui sont également peu développées, font état d'un détachement surprenant à l'égard de la question de la division du contrat. Ainsi Placentin, vers 1165, ne donne dans sa Summa Institutionum aucune définition du contrat, ni même n'emploie le terme de services, alors qu'il énonce dans sa Summa Codicis une définition bipartite du contrat entre la chose et les services dès les premières lignes du titre consacré à la location. La comparaison entre la Summa Institutionum et la Summa Codicis d'Azon, rédigées vers 1210, indique le même phénomène.

De nombreux abrégés au droit romain présentent une vision unitaire centrée sur l'usage de la chose, ce qui vient conforter l'idée que les ouvrages courts privilégient d'autres aspects que la partition du contrat. Le Brachylogus, abrégé de la région d'Auxerre rédigé vers 1166$1167^{30}$, peut se ranger comme la Summa Justiniani est in hoc opere et la Lectura Vindobonensis parmi les ouvrages définissant le contrat sur le fondement exclusif de l'usage de la chose. Dans le titre De locatione et conductione, composé de trois courts paragraphes, la location est d'abord rapprochée de la vente, puis elle en est différenciée : la location ne traite pas de la propriété de la chose, mais de son usage ${ }^{31}$. Les obligations du bailleur sont aussi abordées sous l'angle de l'usage de la chose, puisque le bailleur est tenu de remettre au preneur l'usage de la chose ${ }^{32}$. Le seul exemple évoqué dans le Brachylogus relève exclusivement de la location de la chose, puisqu'il concerne le fermier qui n'aurait pas

sed eo nomine prescriptis verbis actio datur, eo quod innominatus contractus est, et incertum exigitur » (op. cit., p. 128).

${ }^{30}$ GOURON, André. «L'auteur du 'Brachylogus': un compagnon de Thomas Becket en exil ? ». A Ennio Cortese. t. II. Roma: Il Cigno, 2001, p. 169.

${ }^{31}$ Brachylogus, III, 14, 1: "Ita locationis et conductionis differunt tamen, quia in venditione de dominio tradendo agitur; in locatione vero non de dominio rei, sed de usu agitur. » (éd. BÖCKING, E. Berolini, 1829, réimpr. anast. Goldbach, 2002, p. 99, 1. 10-13).

${ }^{32}$ Brachylogus, III, 14, 2 : «Locator vero conductori conducti actione ad usum rei tradendum. » (op. cit., p.100, 1. $1-2)$.

Cadernos do Programa de Pós-Graduação em Direito PPGDir./UFRGS | Edição Digital | Porto Alegre | Volume X | Número 3 | 2015 | P. 65 -89 
acquitté pendant deux ans le loyer dû au bailleur ${ }^{33}$. L'Epitome "Exactis regibus », abrégé anglo-normand composé entre 1150 et $1175^{34}$, livre une remarque procédurale également fondée sur l'usage de la chose par le preneur : ce dernier fait valoir son action en justice pour qu'il puisse utiliser la chose qu'il a prise en location ${ }^{35}$. Une impossibilité d'utiliser la chose constituerait le cas le plus évident de recours à une action en justice du preneur contre le bailleur. D'autres ouvrages font partie de ces collections d'abrégés qui développent des exemples exclusivement basés sur l'usage de la chose, sans toutefois présenter de définition. Le degré de garde de la chose dans la location est estimé en comparaison avec le dépôt et le commodat au chapitre «De la chose louée » des Exceptiones Petri legum Romanorum ${ }^{36}$, seul chapitre de cet ouvrage consacré à la location, et l'auteur du Liber iuris florentinus considère uniquement la location dans le chapitre relatif à l'action en revendication ${ }^{37}$.

Le cas de la location de services est passé sous silence dans ces ouvrages synthétiques, cela ne signifie pas pour autant qu'elle est exclue. Ceci est d'autant plus visible dans les textes qui s'appuient uniquement sur des exemples précis de location de chose, puisque ces développements particuliers n'excluent en rien d'autres types de location. La présentation unitaire du contrat n'est pas non plus une règle absolue des abrégés de droit romain. Dans le Liber pauperum, ouvrage de synthèse du droit romain de la seconde moitié du XII ${ }^{\mathrm{e}}$ siècle, l'auteur, Vacarius, base son raisonnement à propos d'un fragment du Digeste sur la différence entre la location de chose et la location de service, qu'il formule comme tels ${ }^{38}$, et évoque même l'ouvrage à plusieurs reprises.

Les Glossae ad Exceptiones Petri font figure d'exception dans ce tableau des conceptions exclusivement unitaires basées sur l'usage de la chose. La location y fait l'objet

\footnotetext{
${ }^{33}$ Brachylogus, III, 14, 3 : «Et conductor si quidem colonus sit et per continuum biennum debitam pensionem locatori non solverit, non solum locati et Serviana tenetur, sed etiam ius locationis amittit, nisi communis sterilitas regionis et improvisa acciderit. » (op. cit., p.100, 1. 4-8).

${ }^{34}$ LANGE, Hermann. Römisches Recht im Mittelalter. t. I. Die Glossatoren. op. cit., §. 50, p. 422-423.

35 Epitome «Exactis regibus », IX, 32 : «Actione vero conducti experiendum est conductori, ut ei liceat uti re quam conduxit. In has quoque actiones venit quod speciali pacto convenit. » (éd. CONRAT, M. Berlin, 1884, réimpr. anast. Aalen, 1965, p. 131, 1. 6-7).

${ }^{36}$ Exceptiones Petri legum Romanorum, II, 23 : « De re locata. In re locata non tam levis custodia adhibenda est ut in deposita, neque tam gravis ut in commodata, sed mediocris, id est talis qualem bonus paterfamilias in re sua habere solet. » (éd. MOR, C. G. Scritti giuridici preirneriani. t. II. Milano, 1938 [Orbis romanus, t. X], p. 112).

${ }^{37}$ Liber juris florentinus, IV, 16, 4 : «Utilis datur superficiario et ei qui conductum habet vectigalem agrum a municipio et ei qui rem ad prolixum tempus conduxit et forte eadem equitate omni conductori infra tempus conductionis. » (éd. CONRAD, M. Das florentiner Rechtsbuch. Berlin, 1882, p. 82, 1. 32-33, p. 83, 1. 1-2).

${ }^{38}$ VACARIUS. Liber Pauperum, IV, 51 (ad D., 19, 2, 15, 6) : « Multum interest an res vel opera locetur » (éd. ZULUETA, F. DE. The Liber Pauperum of Vacarius. London, 1927 [Publications of the Selden Society, 44], p. 155).
}

Cadernos do Programa de Pós-Graduação em Direito PPGDir./UFRGS | Edição Digital | Porto Alegre | Volume X | Número 3 | 2015 | P. 65 -89 


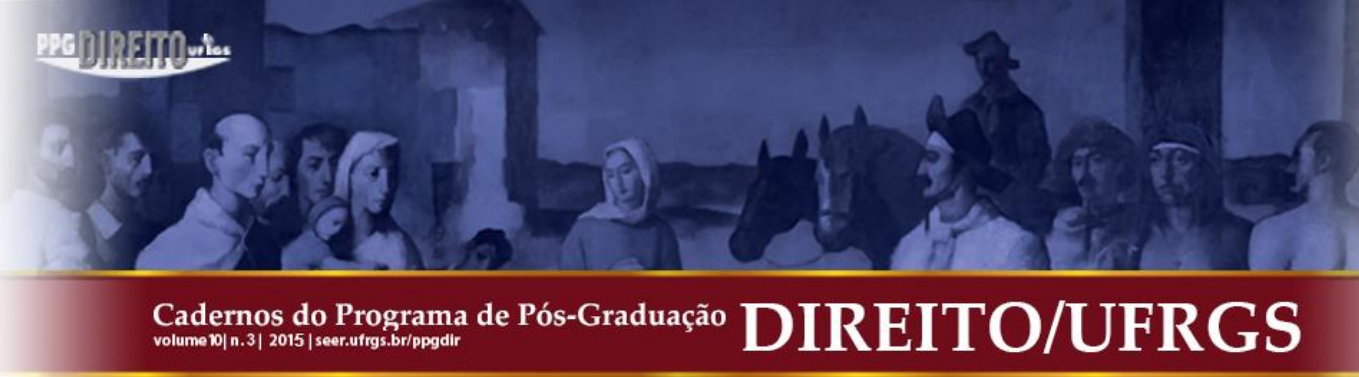

d'une définition selon laquelle il y a location par la partie qui transfère, mais il y a prise en location de la partie qui reçoit ${ }^{39}$. Les mots chose, service ou ouvrage ne sont pas employés, ni même les termes d'usage ou de jouissance, mais l'aspect synallagmatique du contrat et les obligations des parties fondent cette définition unitaire particulière. Cette exception met d'autant plus en relief le caractère fondamental de l'usage de la chose dans le contrat.

\subsection{Les présentations unitaires fondées sur l'usage de la chose, ouvertes à d'autres locations}

Certains ouvrages font de l'usage de la chose la pierre angulaire de leur réflexion sur la location, tout en ouvrant le contrat à la location de services et même d'ouvrage. La Glossa Londinensis, glose aux Institutes composée vers 1215-1220 en Angleterre ${ }^{40}$ en offre l'exemple le plus frappant. Une première définition de la location, puis de la prise en location sont données au regard de l'usage de la chose. La location se caractérise ainsi par le transfert d'une chose pour son usage, et la prise en location, par l'acceptation d'une chose en usage ${ }^{41}$. Ces définitions rappellent celles de la Lectura Vindobonensis par leur balancement entre l'objectif d'utiliser la chose dont le bailleur dit répondre, ad usum, et le caractère passif de l'utilisation par le preneur, in usu. Ces deux ouvrages appartiennent au genre des gloses aux Institutes, ce qui confirme que les ouvrages de synthèse privilégient l'aspect unitaire du contrat fondé sur l'usage de la chose. L'auteur de la Glossa Londinensis ne s'en tient pas toutefois à cette première définition, puisque les termes de services et d'ouvrage, associés à ceux de location et de prise en location, font ensuite irruption dans une autre glose. Selon le texte de cette dernière, quand quelqu'un loue ses services, il est tenu par l'action de prise en location, mais quand quelqu'un prend en location un ouvrage à faire, il est tenu par l'action de location et non par l'action de prise en location ${ }^{42}$. En d'autres termes, le bailleur de ses

39 Gl. ad Exceptiones Petri, 135, 13 : «Locatio fit ex parte dantis, conductio vero ex parte accipientis. » (éd. FITTING, H. Halle, 1874, p. 52).

40 LEGENDRE, Pierre. «Recherches sur les commentaires pré-accursiens ». Tijdschrift voor Rechtsgeschiedenis. t. XXXIII (1965), p. 360 [8].

${ }^{41}$ Glossa Londinensis, 101 (III, XXIV, pr.) : «Locacio est res ad usum data cum diffinicione certe mercedis. Conduccio est res in usu accepta cum mercede consti(tu)ta (éd. LEGENDRE, P. « Recherches sur les commentaires pré-accursiens ». op. cit., p. 381 [29]).

${ }^{42}$ Glossa Londinensis, 475 (III, XXIV) : « Nota quando quis locat operas suas tenetur conducti ; quando vero conducit opus faciendum tenetur locati et non conducti. Locat quis operas suas certa mercede singulis diebus pro 


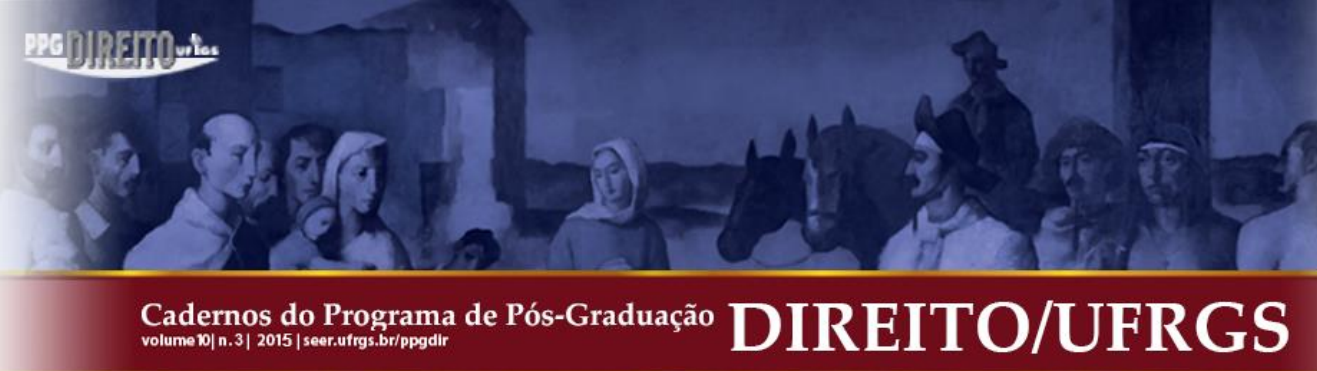

services est soumis à l'action du preneur, tandis que le preneur d'un ouvrage est tenu par l'action du bailleur. L'inversion entre ces deux figures contractuelles doit être remarquée : on est bailleur de ses services, mais on est preneur d'un ouvrage à faire. Cette assertion étonnante n'est pas à proprement parler une définition. Elle pose toutefois les schémas des relations entre les parties, qui correspondent à ceux des opérations qui seront appelées ultérieurement locatio conductio operarum (quand quelqu'un loue ses services), et locatio conductio operis (quand quelqu'un prend en location un ouvrage à faire), la même inversion y est présente. L'exemple de la Glossa Londinensis montre qu'un auteur présentant une définition clairement unitaire du contrat ne s'interdit pas moins de faire référence à d'autres possibilités de location. Cette source questionne le contrat : l'auteur considère-t-il pour autant que la location de services ou la location d'ouvrage constituent des branches à part entière du contrat de location? Les formulations «quand quelqu'un loue ses services » et « quand quelqu'un prend en location un ouvrage à faire » sont limpides, ce qui tend à les détacher de la location de chose. Elles sont encore mises en parallèle, comme si elles formaient deux hypothèses distinctes. De surcroît, le régime juridique de la location de services est différencié de celui de la location d'ouvrage au sein de la même glose ; c'est ainsi qu'une rémunération certaine est établie au jour le jour pour quelqu'un qui loue ses services, et qu'une rémunération est fixée pour l'ensemble de l'ouvrage à faire lorsque quelqu'un prend en location un tel ouvrage. Fautil alors comprendre que la définition de la location s'attache à ce qui semble le plus essentiel dans le contrat, à savoir la location de chose en tant qu'entité distincte, délaissant d'autres locations possibles ? Dans ce cas, le contrat serait déjà non seulement implicitement scindé, mais encore hiérarchisé entre la location de chose et d'autres locations. Une autre hypothèse consiste à considérer que la location de chose a une valeur générale, et forme une unité qui comprend d'autres locations. La location de services et la location d'ouvrage seraient alors des genres de locations de chose, comme si les services et l'ouvrage étaient entendus dans le vocable «chose ». Une autre possibilité, plus décevante, serait d'admettre que la définition de la location omet purement et simplement la location de services ou d'ouvrage. Ceci signifierait cependant que la location de chose revêt une sorte de primauté inconsciente sur d'autres formes de location, et renverrait à la première hypothèse. Il est encore possible de penser que toute location suppose la remise d'une chose, à laquelle peuvent éventuellement 
s'ajouter la prestation de services ou la réalisation d'un ouvrage. Le cas de la Glossa Londinensis n'est pas isolé, puisqu'une même ouverture à d'autres locations se manifeste dans d'autres sources à présentation unitaire, les différentes versions du Lo Codi. Les mêmes hypothèses leur sont applicables, à défaut de pouvoir trancher en faveur de l'une d'entre elles.

La version du Lo Codi en latin, dont la datation est estimée à $1176^{43}$, et les versions postérieures en dauphinois et castillan commencent toutes par une définition centrée sur la chose, qui est vite rectifiée par l'exemple de quelqu'un qui loue son service ${ }^{44}$. Ces trois versions du Lo Codi ont pour point commun de se fonder sur la location de la chose dans le cadre d'une définition. L'idée d'usage n'y est pas exprimée en tant que telle. Dans la version en latin, il est précisé que la chose est transférée «aux fins de moudre ou d'habiller ». La formulation de but fait écho à l'obligation du bailleur de permettre l'usage de la chose. S'agitil pour le bailleur de louer une chose -un moulin, un atelier- dont le preneur doit pouvoir avoir l'usage afin de réaliser des activités qui seraient indépendantes d'une location? Dans ce cas, la location est strictement une location de chose. Alternativement, il serait possible de voir dans cette opération la mise à disposition d'une chose à l'usage du preneur afin qu'il réalise des activités, qui feraient cette fois l'objet d'une location. La définition apparemment unitaire est donc en elle-même sujette à diverses interprétations.

Après cette définition, ces trois textes se poursuivent par une ouverture à la location d'un service, exprimée sous la forme « si quelqu'un loue son service » ou « si quelqu'un loue ses services ». Le cas d'un copiste vient illustrer cette location. Cet exemple du copiste est

\footnotetext{
43 WEIMAR, Peter. «Die legistische Literatur der Glossatorenzeit». In: COING, H. (ÉD.). Handbuch der Quellen und Literatur der neueren europäischen Privatrechtsgeschichte. t. I. Mittelalter (1100-1500). Die gelehrten Rechte und die Gesetzgebung. München: Beck, 1973, p. 200.

${ }^{44}$ Lo Codi, IV, 69, 2 : «Locacio est quando ego do rem meam alii ad pisionem vel ad vestituram pro pecunia quam aliquis dat michi vel promittit. (...). Similiter si aliquis locat operam suam, ipsa est locatio similiter, sicuti si aliquis scribit michi librum pro pecunia quam sibi do vel promitto. » (éd. FITTING, H. ; SUCHIER, H. Lo Codi. Eine Summa Codicis in provenzalischer sprache aus der Mitte des XII. Jahrhunderts. Erster Teil. Lo Codi in der lateinischen Übersetzung des Ricardus Pisanus. Halle : Max Niemeyer, 1906, p. 139, 1. 28-29 et p. $140,1$. 3-4) ; Lo Codi, IV, 69 : «Loiement est quant eu dono ma chosa a un autro a possession per pecuni que cel me done ou me promet. (...). Atressi si alcuns loie s'oura, icen est loiment, issi coma si alcuns escrit a mi un liuro per pecuni que eu li dono o li prometo. » (éd. ROYER, L.; THOMAS, A. «La somme au Code. Texte dauphinois de la région de Grenoble publié d'après un manuscrit du XIII ${ }^{\mathrm{e}}$ siècle appartenant à la bibliothèque du château d'Uriage ». Notices et extraits des manuscrits de la Bibliothèque nationale et autres bibliothèques. t. 42, Paris, 1932, p. 20, 1. 24-25 et 1. 27) ; Lo Codi, IV, 70, 2 : «Logaçión es quando yo algodo una cosa a otro por aver que $<$ da o que me $>$ me promete. (...) Otrossi si alguno aloga las sus obras aquello es alogaçión, (otrossi) assi como si él escrive un libro por aver que ome le da o ome le promete.» (éd. ARIAS BONET, J. A. Lo Codi en Castellano segun los manuscritos 6416 y 10816 de la Biblioteca nacional. Madrid: Universitad Complutense de Madrid, 1984, p. 216).
} 


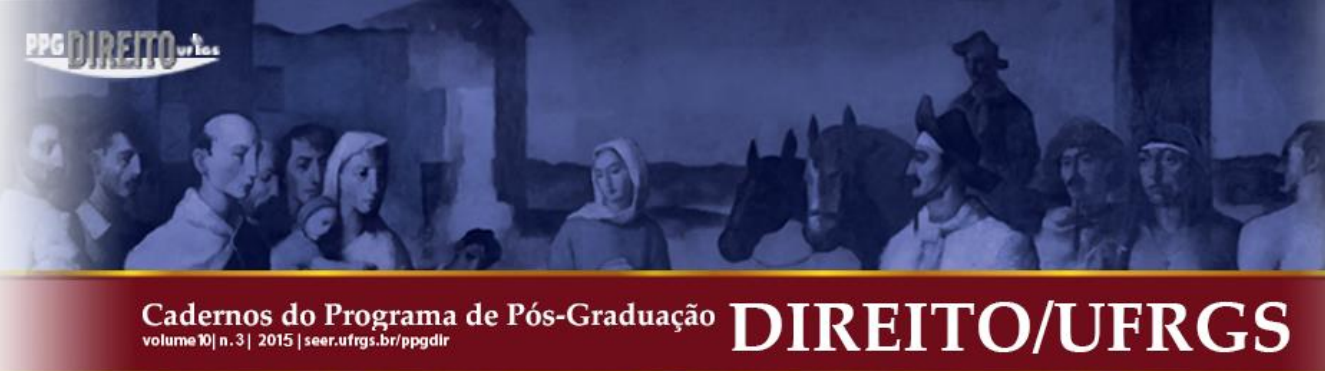

intéressant, puisque sa formulation reprend quasiment les mêmes termes que la définition unitaire. D'après cette dernière, la location est quand «je » transfère ma chose à un autre contre de l'argent qui m'est transféré ou promis. Selon les développements des différentes versions du Lo Codi, quelqu'un écrit un livre contre de l'argent qui lui est transféré ou promis. La comparaison entre la définition et l'illustration conduit d'abord à observer que la contrepartie en argent est un élément unitaire incontestable du contrat. L'attention se porte ensuite sur les deux premières parties des phrases : faut-il conclure que l'entité «écrire un livre » est assimilable au transfert d'une chose à quelqu'un d'autre ? Dans l'affirmative, le service en question, écrire un livre, est-il considéré tout entier comme une chose qui est transférée contre une rémunération, ou, selon une vision plus matérielle, la chose transférée dans le cadre de cette activité est-elle le livre copié lui-même? Ces questions, qui n'appellent pas de réponse définitive, renvoient à nouveau à des hypothèses d'interprétation. La location de chose revêtirait alors une valeur générale, comprenant la location de service par inclusion du service dans la chose, ou alors il faudrait considérer que toute location suppose la remise d'une chose, qu'elle ait fait ou non l'objet de transformations.

La version provençale du Lo Codi paraît plus complète que les autres versions. Une annonce de plan qui distingue clairement la location de chose et la location de services est d'abord faite ${ }^{45}$, puis le même contenu que celui des autres versions apparaît ${ }^{46}$. Quelle est la portée de cette présentation? En lien avec cette interrogation, pourquoi les autres versions du Lo Codi, toutes postérieures à cette version provençale rédigée en 1160 et $1170^{47}$, ne la reprennent-elles pas? La nature d'annonce de plan du texte provençal interdit de lui attribuer une valeur de définition bipartite du contrat entre la chose et les services. Il faut donc à nouveau se contenter de conjectures. Les autres versions pourraient avoir fait l'économie de cette précision d'ordre didactique, indépendamment de la signification véritable qu'elle pourrait revêtir. Il serait ensuite envisageable que l'annonce corresponde à une vision bipartite

\footnotetext{
${ }^{45}$ Lo Codi, IV, 68 : « Aici ditz, cals dreiz es d'aquelas causas que om donna a loguer o d'aquel omen que loga sas obras. » (éd. DERRER, F. Lo Codi. Eine Summa Codicis in provenzalischer Sprache aus dem XII. Jahrhundert. Die provenzalische Fassung der Handschrift A (Sorbonne 632). Zurich: Juris Druck, 1974, p. 100).

${ }^{46}$ Lo Codi, IV, 68, 2 : «Logados es aco, cant eu logui una causa ad autre per auer qu'el m'en dona o qu'el m'en promet. (...). Arregers si alcuns om loga las soas obras, aco es logados eissament, si cum es, si el escrius un libre per auer que om lui done o lui promes. » (éd. DERRER, F. Lo Codi. Eine Summa Codicis in provenzalischer Sprache aus dem XII. Jahrhundert. op. cit., p. 101).

${ }^{47}$ GOURON, André. «Le manuscrit 632 de la Sorbonne : à la convergence des droits savants en pays d'Oc ». Mélanges de la Bibliothèque de la Sorbonne 6. Paris, 1985, p. 13, réimpr. in Droit et coutume en France aux XII et XIII $I^{e}$ siècles, [CS, 422], Adershot, Variorum, 1993, IX.
} 


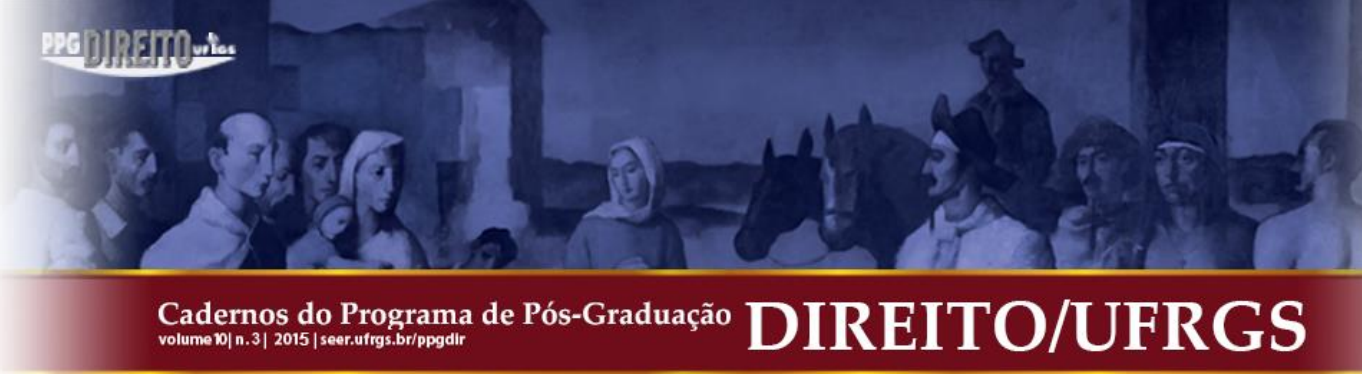

du contrat que les versions postérieures auraient reconnue comme telle et considérée comme une évidence. Pour concilier cette possibilité avec la définition unitaire présente dans toutes les versions du Lo Codi, il faudrait admettre que le contrat de location se définisse avant tout par la location de chose, mais que la location de services s'en distingue. Il est enfin possible de considérer que le texte provençal ne fait pas état d'une bipartition aboutie du contrat mais la préfigure. Le contrat reste défini par la chose, il faut alors revenir aux hypothèses déjà envisagées au sujet du copiste : les services seraient un genre de chose, ou les services viendraient se greffer à la remise d'une chose dans une location de chose.

Les conclusions a minima de cette étude de sources présentant des définitions centrées sur l'usage de la chose peuvent être que les services, ou même la réalisation d'ouvrage, se manifestent toutefois dans ce cadre unitaire. D'autres sources, explicites, autorisent davantage de certitudes, qui s'arrêtent cependant à la bipartition du contrat.

\section{LES DÉCLINAISONS DU CONTRAT DE LOCATION}

Certaines définitions révèlent sans ambiguïté une partition du contrat entre la location de chose et la location de services. La location d'ouvrage apparait dans certains écrits, mais la question de son indépendance des autres locations, en particulier de la location de services, reste entière.

\subsection{Les cas explicites de bipartition du contrat : la chose et les services, la chose et la personne}

Lorsqu'une partition du contrat est exprimée dans le cadre d'une définition, il s'agit toujours de partager le contrat entre la location de chose et la location de services. La définition précitée de la Summa Vindobonensis est peut-être l'une des premières en ce sens, avec celle de la Summa Trecensis ${ }^{48}$, une somme au Code rédigée en Provence entre 1135 et 1140, dont l'auteur, Géraud, a été identifié par André Gouron en $1984^{49}$. Les définitions de la Summa Vindobonensis et de la Summa Trecensis sont quasiment identiques, comme l'avait

48 Summa Trecensis, IV, 57, 2 : «Locatio quidem est cum usum rei mee, vel operas meas pro mercede tibi do » (éd. FITTING, H. Summa Codicis des Irnerius. Berlin: Guttentag, 1894, p. 131, 1. 24, et p. 132, 1.1).

${ }^{49}$ GOURON, André. «L'auteur et la patrie de la Summa Trecensis ». Ius commune. t. 12 (1984), p. 28.

Cadernos do Programa de Pós-Graduação em Direito PPGDir./UFRGS | Edição Digital | Porto Alegre | Volume X | Número 3 | 2015 | P. 65 -89 


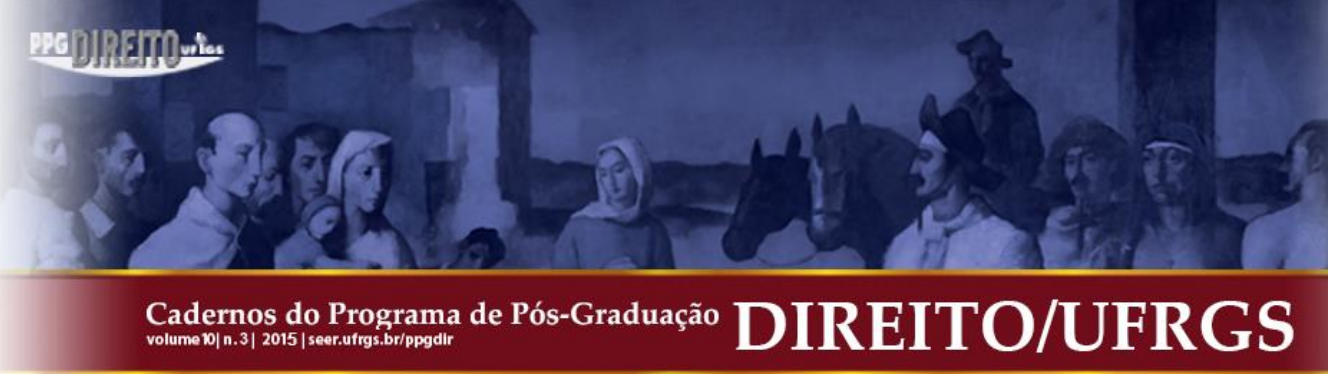

souligné Gerhard Otte ${ }^{50}$. Ces définitions proposent qu'il y ait location lorsqu'il y a concession de l'usage d'une chose ou de services. L'usage concerne uniquement la chose, la même précaution ne s'applique pas aux services.

Placentin, entre 1160 et 1170, donne dans sa somme au Code une définition bipartite très semblable à celle de la somme au Code d'Azon, vers 1208-1210. «Or, la location consiste dans la remise d'une personne ou d'une chose pour un usage, avec l'établissement d'une rémunération certaine à chiffrer en argent $»^{51}$, d'après Placentin, et «la location consiste dans la remise d'une personne ou d'une chose pour un usage, quand il y a accord sur une rémunération à chiffrer en argent $»^{52}$, selon Azon. Le premier réflexe, à la comparaison de ces deux définitions, est de songer à une influence directe de Placentin sur Azon. Cependant, les deux auteurs pourraient avoir pour source commune les enseignements de Bulgarus. Placentin a en effet suivi à Bologne les cours de Bulgarus ${ }^{53}$, qui était aussi le maître de Jean Bassien $^{54}$, dont Azon était l'élève ${ }^{55}$. En outre, Azon a été probablement davantage influencé par son maître Jean Bassien, qu'il citait souvent et contredisait peu ${ }^{56}$, que par Placentin.

Dans ces deux définitions, le mot «services » est remplacé par celui de «personne », et l'usage concerne à la fois la chose et la personne. La substitution du terme services par celui de personne ainsi que l'emploi du mot usage à propos de la personne ne sont pas anodines, et conduisent à des questions relatives au traitement de la personne à la fois par Placentin et Azon, et par les glossateurs en général. Les définitions de Placentin et Azon permettent certes d'identifier une différenciation claire entre la personne et la chose. Faut-il en conclure que les auteurs des autres définitions qui utilisent le terme services, ou qui sont centrées sur la chose, ne pensent pas une telle différence entre la chose et la personne? A contrario, Placentin et Azon assimilent-ils complètement la personne aux services qu'elle peut rendre ? Si leurs définitions ont pour effet de distinguer une personne d'une chose, ne réifient-elles pas la personne en évoquant son usage? La mention de l'usage au sujet de la personne pourrait

\footnotetext{
${ }^{50}$ OTTE, Gerhard. Dialektik und Jurisprudenz. Untersuchungen zur Methode der Glossatoren. Frankfurt am Main, 1971 (Ius Commune, Sonderhefte, t. I), p. 112.

${ }^{51}$ PLACENTINUS. Summa Codicis, IV, 65: «Est autem locatio personae reive ad usum facta concessio, mercede certa constituta in pecunia numeranda », Moguntiae, 1536, réimpr. anast. Torino, 1962, p. 189.

${ }^{52}$ AZO. Summa Codicis, IV, 65 : «Est autem locatio persone reive ad usum facta concessio, mercede in pecunia numeranda et conventa », Papie, 1506, réimpr. anast. Augustae Taurinorum, 1966 (Corpus Glossatorum Juris Civilis, t. II), p. 170, colonne 1.

${ }^{53}$ LANGE, HeRmanN. Römisches Recht im Mittelalter. v. I. Die Glossatoren. op. cit., p. 208.

${ }^{54}$ Ibid., p. 255.

${ }^{55}$ Ibid., p. 216.

${ }^{56}$ Ibid., p. 256.
}

Cadernos do Programa de Pós-Graduação em Direito PPGDir./UFRGS | Edição Digital | Porto Alegre | Volume X | Número 3 | 2015 | P. $65-89$ 


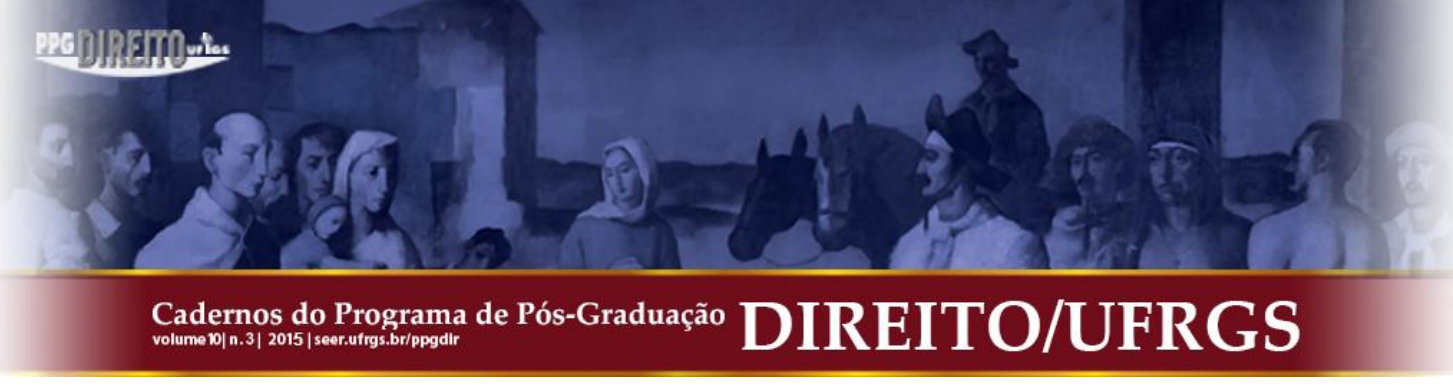

avoir pour fonction de nuancer la distinction entre chose et personne.

La question de l'identification de la personne et du service ne reste pas totalement en suspens. Dans la même somme au Code, Placentin évoque la rémunération du bailleur «quand la personne est louée, ou le service de la personne ${ }^{57}$. La conjonction « ou » (sive) pourrait indiquer une distance entre la personne et le service, mais l'emploi de l'expression « la personne est louée », illustrée par les exemples du paysan et du secrétaire, incline au contraire à penser que Placentin assimile la personne et son service.

Des formulations, dont certaines datent du début de l'école des glossateurs, expriment une partition du contrat entre la location de chose et celle de service. Elles ne sont pas des définitions à proprement parler, mais révèlent que leurs auteurs envisagent au moins une telle partition du contrat entre la chose et les services. Dans la première moitié du XII siècle, Irnerius distingue la location de chose de la location de services ${ }^{58}$. Le régime juridique de chaque cas est ensuite exposé. Il est à noter que cette distinction n'est nullement contradictoire avec la mention par ailleurs de l'obligation du bailleur de répondre de la jouissance de la chose ${ }^{59}$. Vacarius, dans la seconde moitié du XII ${ }^{\mathrm{e}}$ siècle en Angleterre, opère aussi une distinction entre la location de chose ou de service, dans une présentation précitée. La partition du contrat peut aussi s'illustrer dans l'analyse de cas. La Collectio Codicis Chisiani 218, un ouvrage appartenant au genre de la dispute scolaire de la fin du XII ${ }^{\mathrm{e}}$ siècle ${ }^{60}$, fait état de la question de la location perpétuelle de services par un homme libre ${ }^{61}$.

Cette séparation du contrat entre la location de chose et la location de services peut être approfondie, parfois par les mêmes auteurs, parfois par d'autres.

\subsection{Au-delà de la bipartition du contrat ?}

57 PLACENTINUS. Summa Codicis, IV, 65 : «Distinguitur ita locatio: locatur alias persona, ut fossoris, et excerptoris, alias res, ut ager, domus, equus. Et certe ubi locatur persona, sive personae opera, locator debet recipere personae mercedem $»$ (op. cit., p. 190).

58 IRNERIUS. Dist. Par. 4603, 30 : «Locat quis quandoque rem suam, quandoque operas suas » (éd. PESCATORE, G. Die Glossen des Irnerius. Greiswald, 1888, réimpr. anast. Frankfurt am Main, 1968. t. II, p. 70).

${ }^{59}$ ID. Gl. ad D., 19, 2, 15, 2, Ex conducto, $\mathrm{V}^{\text {is }}$ Vis tempestatis : " Quamvis locator teneatur ad hoc ut rei fructum prestet, si tamen fructus fortuito vel vi interciderit, excusatur ita tamen ut et ipse careat mercede, quod in venditore non ita est. Is enim precium feret licet res interierit » (éd. BESTA, E. L'opera d'Irnerio. Torino, 1896, t. II, p. 198).

${ }^{60}$ LANGE, Hermann. Römisches Recht im Mittelalter. t. I. Die Glossatoren. op. cit., p. 148.

61 Collectio Codicis Chisiani 218, §. 81: «An liber homo operas suas in perpetuum locare possit? Differunt. » (éd. HÄNEL, G. Dissensiones dominorum sive iuris romani interpretum. Lipsiae, 1834, réimpr. Aalen, 1964, p. 181).

Cadernos do Programa de Pós-Graduação em Direito PPGDir./UFRGS | Edição Digital | Porto Alegre | Volume X | Número 3 | 2015 | P. 65 -89 


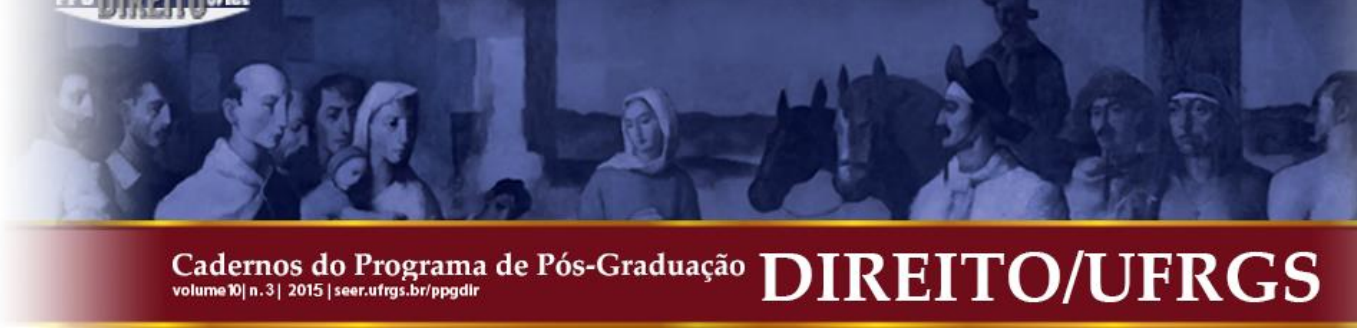

Il serait abusif d'évoquer une tripartition claire du contrat. Néanmoins, le mot opus, ouvrage, apparaît dans certaines sources, de manière plus ou moins identifiée et plus ou moins récurrente. Le terme d'ouvrage est parfois utilisé en relation avec celui de services, sans qu'il soit possible de déterminer si les deux termes sont individualisés. L'emploi d'opus au sujet de la location se retrouve très tôt, dans les gloses au Digeste d'Irnerius. Le cas de la location d'un entrepôt qui fait l'objet d'une garde y est exposé ${ }^{62}$. Ce cas est analysé par Irnerius comme une location de chose dans laquelle est effectué un service, selon une conception bipartite du contrat partagé entre la location de chose et de services. Il est ensuite expliqué que « celui qui prend en location des ouvrages est appelé au contraire bailleur d'ouvrage ». Cette phrase peut surprendre, puisque le bailleur (locator) y est dit prendre en location (conducit). Faut-il y voir de manière globale la description du renversement de la qualification des parties entre la location de services et la location d'ouvrage, dont l'inversion serait soulignée par Irnerius ( $e$ contrario) ? La relation entre cette assertion et l'opération de location d'entrepôt faisant l'objet d'une garde n'est pas suffisamment claire pour en avoir la certitude.

Les services ne sont pas non plus parfaitement distingués de l'ouvrage dans la glose ordinaire. Il faut ici distinguer la glose ordinaire au Digeste et la glose ordinaire au Code.

La location d'un ouvrage à faire est certes évoquée sans détour dans la glose ordinaire au Digeste ${ }^{63}$. Il y est ainsi explicitement question de louer un ouvrage à faire. Les parties font aussi l'objet d'une identification : «il» représente la partie qui loue, le bailleur, et «moi », celle qui reçoit la rémunération. Le preneur réalise donc l'ouvrage, ce qui distingue cette opération de la location de service, dans laquelle le bailleur effectue les services. Le régime juridique est même précisé, puisque le preneur doit recevoir sa rémunération pour tout l'ouvrage effectué.

Cependant, les rapports entre les services et l'ouvrage sont présentés de manière beaucoup plus indistincte dans la glose ordinaire au Code. La constitution qui sert de base à la

\footnotetext{
${ }^{62}$ IRNERIUS. Gl. ad D., 19, 2, 57, Qui domum : «Ita et hoc dici solet me conducere eam rem in quam impendo meam operam, cum possim et alii locare idem faciendum. Item tibi loco horreum ut tibi non tam ad te et rei quam ad metum earum rerum custodia pertinet. Prestat igitur custodiam non tantum conductor, sed interdum etiam locator cum idem et conductor dicatur: et e contrario qui conducit opera locator operis vocitatur » (éd. BESTA, E. L'opera d'Irnerio. op. cit., p. 202).

${ }^{63}$ ACCURSIUS. Gl. ord. ad D., 19, 2, 33, Si fundus, Vo Locasses : «Locavit ergo opus ad faciendum, ut mihi pro toto opere praestaret mercedem »(Glossa in Digestum vetus, [Corpus Glossatorum Juris Civilis, t. VII], Venetiis, 1488, réimpr. anast., Augustae Taurinorum, 1969, fol. 290 va).
} 
réflexion évoque la question de la dette de la personne qui a pris en location des édifices à construire $^{64}$. Dans cette constitution, il n'est pas complètement évident que cette personne soit chargée de réaliser les bâtiments. Cette partie pourrait tout aussi bien, en l'absence de contextualisation, être un client qui prendrait en location des édifices à construire. Il est clair, néanmoins, que cette personne est preneur, puisqu'elle prend en location. Or, dans la glose ordinaire à cette constitution, cette personne qui réclame sa dette est assimilée à un artisan, qui est lui-même qualifié de bailleur d'un service ${ }^{65}$. La même personne est donc preneur dans la constitution, et bailleur dans la glose au Code. La comparaison des actions préconisées par la constitution et sa glose est révélatrice de la requalification de la partie concernée. Le preneur de la constitution poursuit sa dette par une action de prise en location, ex conducto, alors que selon la glose, l'artisan bailleur peut agir par une action de location-prise en location, ex locato et conducto, comme si la simple mention de l'action ex locato avait rendu trop évidente cette requalification. Une autre glose complète la réflexion sur ce cas en posant la question de la date à laquelle le bailleur de services peut réclamer une rémunération ${ }^{66}$. La réponse est surprenante, puisqu'il est dit que la rémunération peut être réclamée à la fin de l'ouvrage quand on a pris en location à son risque. Il peut d'abord être observé que le bailleur de ses services peut réaliser un ouvrage. Le rétablissement du verbe «prendre en location », alors même que le bailleur de services loue, est aussi à souligner : est-ce parce qu'il est question d'un ouvrage que ce verbe est employé ? Cet exemple tiré de la glose ordinaire n'est pas sans rappeler l'analyse de Roberto Fiori, selon laquelle la location d'ouvrage a pu être considérée jusqu'au début du XVII ${ }^{\mathrm{e}}$ siècle comme une location de service dont le prestataire assumerait la responsabilité et le risque ${ }^{67}$.

L'ouvrage est aussi évoqué dans certaines sources, sans faire l'objet d'une classification. La perception du contrat est particulièrement étagée chez Odofrède, dans la seconde moitié du XIII ${ }^{\mathrm{e}}$ siècle, puisqu'il commence par proposer une définition unitaire du

\footnotetext{
${ }^{64}$ ANTONINUS. C., 4, 65, 2, [a. 213] : «Adversus eos, a quibus extruenda aedificia conduxisti, ex conducto actione consistens eo judicio quod est bonae fidei debitum cum usuris solitis consequeris ».

${ }^{65}$ ACCURSIUS. Gl. ord. ad C., 4, 65, 2, Adversus, $\mathrm{V}^{\circ}$ Conduxisti : " Quod conductio dicitur nomine edificii et eiusdem ratione dominus dicitur locator. Item conductor dicitur dominus ratione eiusdem opere et faber locator ratione eiusdem opere unde faber potest agere ex locato et conducto ad mercedem » (Glossa in Codicem, [Corpus Glossatorum Juris Civilis, t. X], Venetiis, 1488, réimpr. anast., Augustae Taurinorum, 1968, fol. 125 va).

${ }^{66}$ ID. Gl.ord. eod. loc., $\mathrm{V}^{\circ}$ Solitis: «Sed iste qui sic locavit operas, quando poterit petere mercedem? Respondetur finito opere (...) sed illud quando suo periculo conduxit, unde quando non suo periculo conduxisset, posset dici contra » (op. cit., fol. 125 va).

${ }^{67}$ FIORI, ROBERTO. La definizione della «locatio conductio », op. cit., p. 306-307.
} 
contrat centrée sur la chose. Le bailleur répond ainsi de la chose, et le preneur, de la rémunération $^{68}$. Cette définition convient aussi à une location de services, que l'auteur envisage en citant les exemples de l'ouvrier et du secrétaire qui louent leurs services. L'hypothèse d'une location d'ouvrage est implicitement écartée dans cette définition, puisque la partie qui répond de la rémunération est alors le bailleur. Pourtant, Odofrède n'exclut pas par ailleurs la possibilité d'une location d'ouvrage, d'abord parce qu'il identifie le preneur d'ouvrage comme le créancier d'une rémunération. Il évoque ainsi le cas où « je » loue un ouvrage à faire à «tu », qui doit percevoir une rémunération certaine chaque jour ${ }^{69}$. Dans ce schéma, «je » est bailleur et «tu», preneur, est le destinataire de la rémunération, ce qui s'oppose à la définition précitée. Ensuite, Odofrède emploie de manière récurrente la formulation «si je t'ai loué un ouvrage à faire $»^{70}$, avec la variante «si j'ai pris en location un ouvrage à faire $»^{71}$. L'emploi de cette expression construite autour de l'ouvrage à faire, sous la forme figée opus faciendum, correspond à des fragments du Digeste où le terme opus est employé sous sa forme déclinée. L'expression opus faciendum n'est employée qu'une seule fois dans le Digeste ${ }^{72}$. L'usage systématique de cette expression par Odofrède semble révéler la construction de l'opération juridique de location d'ouvrage, alors même qu'elle n'est pas considérée dans le cadre de sa définition du contrat.

La présentation qui identifie le plus les trois hypothèses de contrat est certainement celle $\mathrm{d}^{\prime} \mathrm{Azon}^{73}$. Elle s'insère après la définition bipartite selon laquelle la location consiste dans la

${ }^{68}$ ODOFREDUS. Com. ad C., 4, 65 : « Nam proprie est locator, qui prestat rem. Proprie est conductor, qui prestat mercedem. (...) Sed nos habemus talem considerationem ibi, ut dicamus faber et scriptor proprie dicitur locare operas suas mihi, et dicor ego conducere, qui solvo certum quod pro opera. » (In primam Codicis partem praelectiones, Lugduni, 1552, réimpr. anast. Bologna, 1968, [Opera Juridica Rariora, t. v/1], fol. 257 ra).

${ }^{69}$ ID. Com. ad D., 19, 2, 51, 1, Locavi opus : «In paragrapho isto dicitur : locavi tibi aliquod opus faciendum eo pacto ut tu deberes habere certam mercedem singulis diebus pro illo opere » (In secundam Digesti veteris partem praelectiones, Lugduni, 1552, réimpr. anast. Bologna, 1968, [Opera Juridica Rariora, t. II/2], fol. 120 ra)

${ }^{70}$ ID. Com. ad D., 19, 2, 13, 10, Si in lege operis (op. cit., fol. 115 va) ; ID, Com. ad D., 19, 2, 24, pr., Si in lege (op. cit., fol. $117 \mathrm{vb}$ ) ; ID, Com. ad D., 19, 2, 30, 3, Qui edem (op. cit., fol. $118 \mathrm{vb}$ ) ; ID. Com. ad D., 19, 2, 36, Opus quod adversione locatum est (op. cit., fol. 119 va) ; ID. Com. ad D., 19, 2, 37, Si prius (op. cit., fol. 119 va); ID. Com. ad D., 19, 2, 51, 1, Locavi opus (op. cit., fol. 120 ra) ; ID. Com. ad D., 19, 2, 58, 1, In operis locatione (op. cit., fol. $120 \mathrm{rb}$ ).

${ }^{71}$ ID. Com. ad D., 19, 2, 48, pr., Si cui locaverim (op. cit., fol. $119 \mathrm{vb}$ ).

72 IAVOLENUS. Libro undecimo epistularum, D., 19, 2, 51, 1.

73 AZO. Summa Codicis, IV, 65 : «Ergo si domum haberem et te conventa mercede inhabitare permitto, tu conductor ergo locator dicor ut ff., eodem, Si tibi [D., 19, 2, 5] et lege Cum fundus [D., 19, 2, 3] et infra, eodem, lege III $[D ., 19,2,3]$ et lege Nemo [D., 19, 2, 51, pr.] et lege Conductoris $[D ., 19,2,24,1]$, tuque si operis tuis mercede conventa in re mea utaris diceris locator operarum tuarum, et ego earumdem conductor ut ff., eodem, Qui operas [D., 19, 2, 38, pr.]. Si vero tibi opus faciendum mercede conventa dedero respectu operis ego locator, tu vero conductor diceris ut ff., eodem, Item queritur, §. I [D., 19, 2, 13, pr.], et $\S$. Si gemma $[D ., 19,2,13,5]$ et 


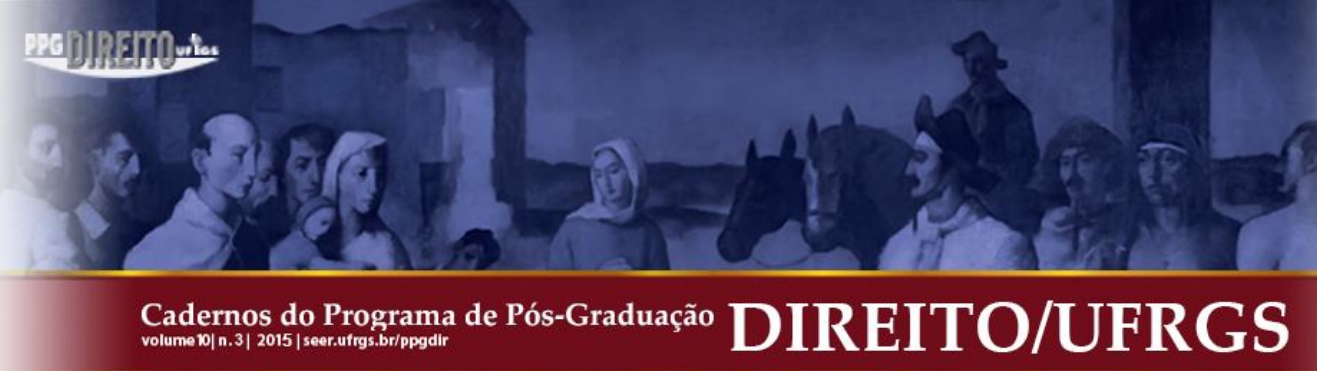

remise d'une personne ou d'une chose pour un usage, ce qui invite plutôt à penser qu'elle vient expliquer la définition sous l'angle de différentes situations concrètes, afin de faciliter la compréhension du lecteur. D'un côté, l'aspect didactique de ce paragraphe se fait jour grâce à plusieurs indices. Cet exposé s'attache en effet tout spécialement à qualifier les parties, bailleur ou preneur. Il s'agit en outre d'une présentation concrète, puisque l'auteur commence par évoquer le cas d'une maison. D’un autre côté, un aspect théorique se manifeste. Trois hypothèses différentes apparaissent ainsi, identifiées par «si »: «si j'ai une maison et je te permets d'y habiter », «si tu fais usage de tes services », «si en revanche je t'ai donné un ouvrage à faire ». De plus, chaque hypothèse est illustrée par un ou plusieurs renvois au Digeste.

La première hypothèse, «si j'ai une maison et je te permets d'y habiter », illustre une location de choses. Le bailleur permet au preneur d'utiliser ce qui est à lui, la maison. Même si Azon ne précise pas qui perçoit la rémunération, il souligne l'existence d'une rémunération convenue, et on peut déduire du sens de l'opération que le bailleur reçoit une rémunération du preneur.

Dans la deuxième hypothèse, «si tu fais usage de tes services », «toi », qui était preneur dans la première hypothèse, devient bailleur parce qu'il propose de réaliser des services au profit du preneur. L'autre partie, «je» qui était bailleur de la chose, devient preneur des services. Il est important de relever que les parties changent de qualification entre les deux hypothèses. La partie qui touche la rémunération n'est à nouveau pas précisée. Il découle de l'opération décrite par Azon que le bailleur, «tu», qui effectue les services, perçoit la rémunération.

La troisième hypothèse, «si en revanche je t'ai donné un ouvrage à faire », envisage la location d'un ouvrage. La partie qui donne l'ouvrage à faire est bailleur, celle qui l'exécute est preneur. Il n'est pas précisé si le bailleur fournit la matière sur laquelle travaille le preneur. Azon insiste au contraire sur l'action de donner l'ouvrage à faire, ce qui invite à penser que le bailleur est identifié par l'ordre qu'il donne, et non par la matière qu'il fournirait éventuellement. L'expression « une fois la rémunération convenue » est à nouveau employée, sans autre précision. Puisque le preneur effectue dans ce cas un ouvrage, il reçoit du bailleur une rémunération. Les allégations se réfèrent aux cas d'un cocher, d'un foulon et d'un

§. Si fullo $[D ., 19,2,13,6]$, et lege Opus $[D ., 19,2,36]$, et quasique diceris, redemptor, ut ff., eodem, Qui insulam, §. Qui edem [D., 19, 2, 30, 3]» (op. cit., p. 170, colonne 1).

Cadernos do Programa de Pós-Graduação em Direito PPGDir./UFRGS | Edição Digital | Porto Alegre | Volume X | Número 3 | 2015 | P. 65 -89 


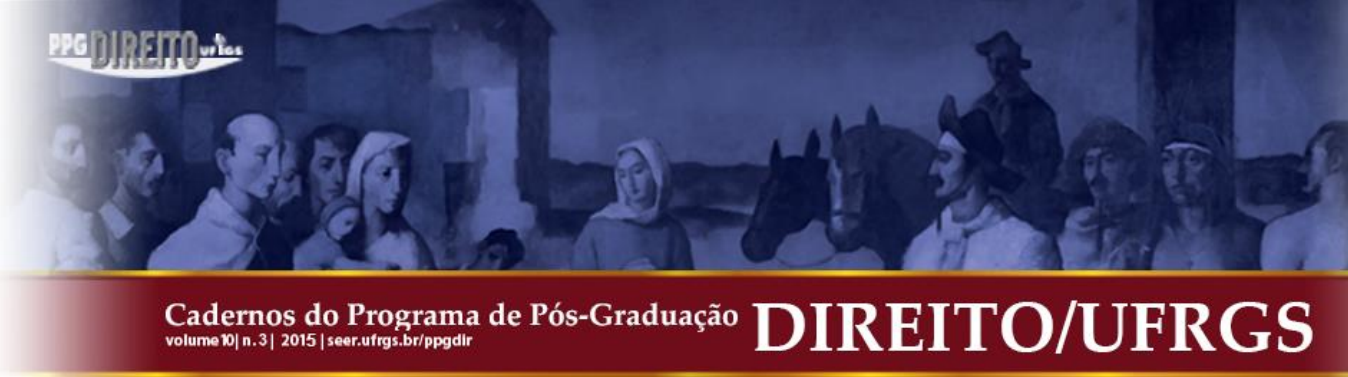

EPITOME «Exactis regibus»(Die.). Mit Anhängen und einer Einleitung: Studien zur Geschichte des römischen Rechts im Mittelalter. In: CONRAT, M. (éd.). Berlin, 1884, réimpr. anast. Aalen, 1965.

EXCEPTIONES Petri legum Romanorum. In: MOR C. G. (éd.). Scritti Giuridici Preirneriani. t. II. Milano, 1938 [Orbis romanus, t. X].

GLOSSA Londinensis ad Institutiones. In : LEGENDRE, P. (éd.). «Recherches sur les commentaires pré-accursiens ». Tijdschrift voor Rechtsgeschiedenis, t. XXXIII (1965), p. 353429.

GLOSSE zu den Exceptiones legum Romanorum des Petrus. In: FITTING, H. (éd.). Halle, 1874.

HEINECCIUS, Johannes Gottlieb. Elementa juris civilis secundum ordinem Institutionum commoda auditoribus methodo. Giessae, ca. 1750.

IRNERIUS. Glossae ad Digestum vetus. In: BESTA, E. (éd.). L'opera d'Irnerio. t. II. Torino, 1896.

In: PESCATORE, G. (éd.). Die Glossen des Irnerius. t. II. Greiswald, 1888, réimpr. anast. Frankfurt am Main, 1968.

LECTURA Vindobonensis super Institutiones. In: PALMIERI, J.B. (éd.). Wernerii summa Institutionum cum glossis Martini, Bulgari, Alberici aliorumque. Bibliotheca Iuridica Medii Aevi. t. I. Additiones. Bononiae, 1914, p. 1-208 (notae).

LIBER juris florentinus. In: CONRAD, M. (éd.). Das florentiner Rechtsbuch, ein System römischen Privatsrechts aus der Glossatorenzeit. Berlin, 1882.

LO Codi. In: ROYER, L.; THOMAS, A. (éd.). «La somme au Code. Texte dauphinois de la région de Grenoble publié d'après un manuscrit du XIII ${ }^{\mathrm{e}}$ siècle appartenant à la bibliothèque du château d'Uriage ». Notices et extraits des manuscrits de la Bibliothèque nationale et autres bibliothèques. t. 42. Paris, 1932. 


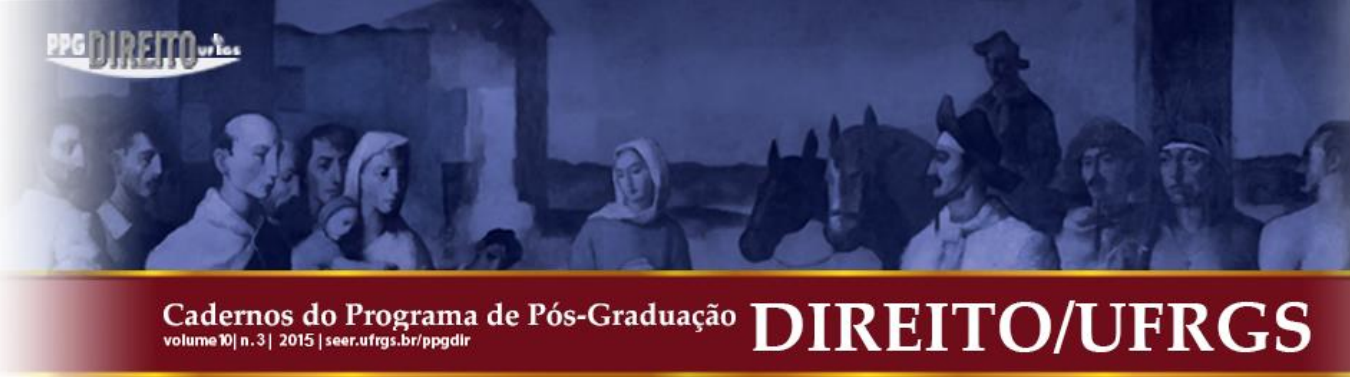

LO Codi. In: DERRER, F. (ÉD.). Eine Summa Codicis in provenzalischer Sprache aus dem XII. Jahrhundert. Die provenzalische Fassung der Handschrift A (Sorbonne 632). Zurich, 1974.

LO Codi. In: FITTING, H.; SUCHIER, H. (éd.). Eine Summa Codicis in provenzalischer sprache aus der Mitte des XII. Jahrhunderts. Erster Teil. Lo Codi in der lateinischen Übersetzung des Ricardus Pisanus. Halle, 1906.

LO Codi en Castellano segun los manuscritos 6416 y 10816 de la Biblioteca nacional. In: ARIAS BONET, J. A. (éd.). Madrid, 1984.

ODOFREDUS DE DENARIIS. In primam Codicis partem complectentem I., II., III., IIII., et V libros praelectiones, Lugduni, 1552, réimpr. anast. Lectura super Codice I, Bologna, 1968 (Opera Juridica Rariora, t. v/1).

. In secundam Digesti veteris partem praelectiones. Lugduni, 1552, réimpr. anast. Lectura super Digesto veteri II, Bologna, 1968 (Opera Juridica Rariora, t. II/2).

PLACENTINUS. Summa Codicis. Moguntiae, 1536, réimpr. anast. Torino, 1962.

SINTENIS, Carl Friedrich Ferdinand. Das practische gemeine Civilrecht. t. II. Das Obligationenrecht. Leipzig, 1847.

SUMMA Institutionum "Iustiniani est in hoc opere» (Ms. Pierpont Morgan 903). In: LEGENDRE, P. (éd.). Frankfurt am Main, 1973 (Jus commune, Sonderhefte, 2).

SUMMA Institutionum Londinensis. In: ZULUETA, F. DE; STEIN, P. (éd.). The teaching of Roman Law in England around 1200. London, 1900 (Selden Society, Suppl. Ser., 8).

SUMMA Trecensis. In: FITTING, H. (éd.). Summa Codicis des Irnerius. Berlin, 1894.

SUMMA Vindobonensis. PALMIERI, J.B. (éd.). Wernerii summa Institutionum cum glossis Martini, Bulgari, Alberici aliorumque. Bibliotheca Iuridica Medii Aevi. t. I. Additiones. Bononiae, 1914, p. 1-208.

VACARIUS. Liber Pauperum. In: ZULUETA, F. DE (ED.). The Liber Pauperum of Vacarius. London, 1927 (Publications of the Selden Society, 44). 


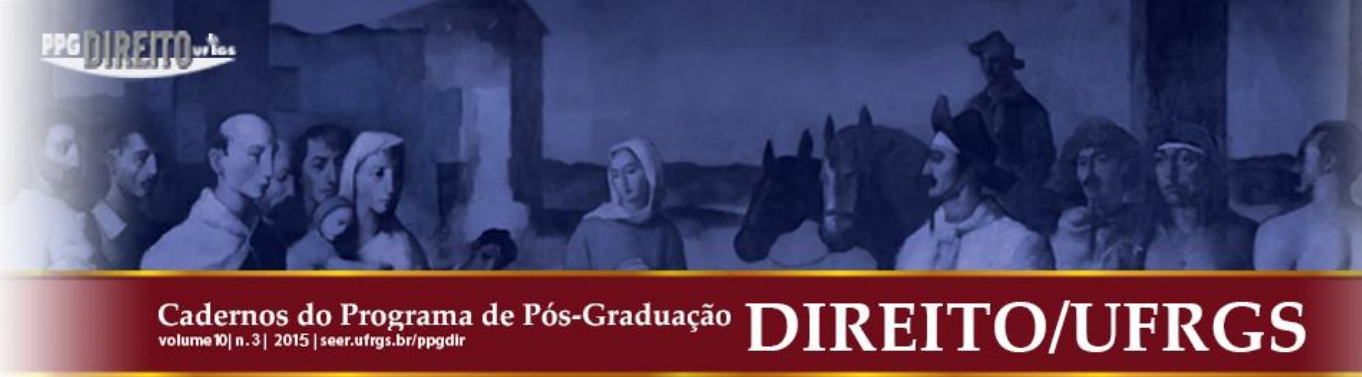

VOET, Johannes. Commentarius ad Pandectas. Hagae-Comitum, 1707.

\section{II.- BIBLIOGRAPHIE}

FIORI, Roberto. La definizione della «locatio conductio». Giurisprudenza romana $e$ tradizione romanistica. Napoli: Jovene, 1999, XI- 410 p.

GOURON, André. «La science juridique française aux $\mathrm{XI}^{\mathrm{e}}$ et $\mathrm{XII}^{\mathrm{e}}$ siècles : diffusion du droit de Justinien et influences canoniques jusqu'à Gratien ». Ius Romanum Medii Aevi I, 4, d-e, Mediolani, Giuffrè, 1978, p. 3-118, réimpr. in Études sur la diffusion des doctrines juridiques médiévales. London: Variorum, 1987 (CS, 264), II.

. «L'auteur du 'Brachylogus' : un compagnon de Thomas Becket en exil ? ». A Ennio

Cortese. t. II. Roma: Il Cigno, 2001, p. 161-173.

. «L'auteur et la patrie de la Summa Trecensis ». Ius commune, t. 12 (1984), p. 1-38.

«Le manuscrit 632 de la Sorbonne : à la convergence des droits savants en pays d'Oc ». Mélanges de la Bibliothèque de la Sorbonne 6, Paris, 1985, p. 6-20, réimpr. in Droit et coutume en France aux XII et XIII siècles, [CS, 422], Adershot, Variorum, 1993, IX.

LANGE, Hermann. Römisches Recht im Mittelalter. t. I. Die Glossatoren. München: Beck, 1997, XXXI- 485 p.

LEGENDRE, Pierre. «Recherches sur les commentaires pré-accursiens ». Tijdschrift voor Rechtsgeschiedenis, t. XXXIII (1965), p. 353-429.

OLIVIER-MARTIN, Félix. «Des divisions du louage en droit romain » . Revue historique de droit français et étranger. t. 15 (1936), p. 419-475.

OTTE, Gerhard. Dialektik und Jurisprudenz. Untersuchungen zur Methode der Glossatoren. Frankfurt am Main, 1971 (Ius Commune, Sonderhefte, t. I), 255 p. 


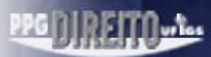

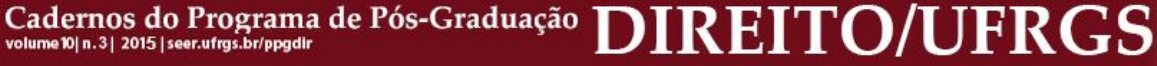

RANIERI, Filippo. «Dienstleistungsverträge: Rechtsgeschichte und die italienische Erfahrung ». In: ZIMMERMANN, R. (ED.). Service contracts. Gesellschaft für Rechtsvergleichung. (Rechtsvergleichung und Rechtsvereinheitlichung, 15). Tübingen: Mohr Siebeck, 2010, p. 1- 41.

WEIMAR, Peter. «Die legistische Literatur der Glossatorenzeit». In: COING, H. (ÉD.). Handbuch der Quellen und Literatur der neueren europäischen Privatrechtsgeschichte. t. I. Mittelalter (1100-1500). Die gelehrten Rechte und die Gesetzgebung. München: Beck, 1973, p. $129-260$.

Submissão: 30/09/2015

Aceito para Publicação: 21/12/2015 


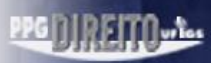

Cadernos do Programa de Pós-Graduação

DIREITO/UFRGS 